\title{
Adding psychosocial therapy to methylphenidate may not improve its effectiveness in stimulant responsive children with ADHD
}

Abikoff H, Hechtman L, Klein RG, et al. Symptomatic improvement in children with ADHD treated with long-term methylphenidate and multimodal psychosocial treatment. J Am Acad Child Adolesc Psychiatry 2004;43:802-11.

\section{Does adding psychosocial therapy to methylphenidate improve effectiveness of treatment for ADHD in stimulant responsive children?}

METHODS

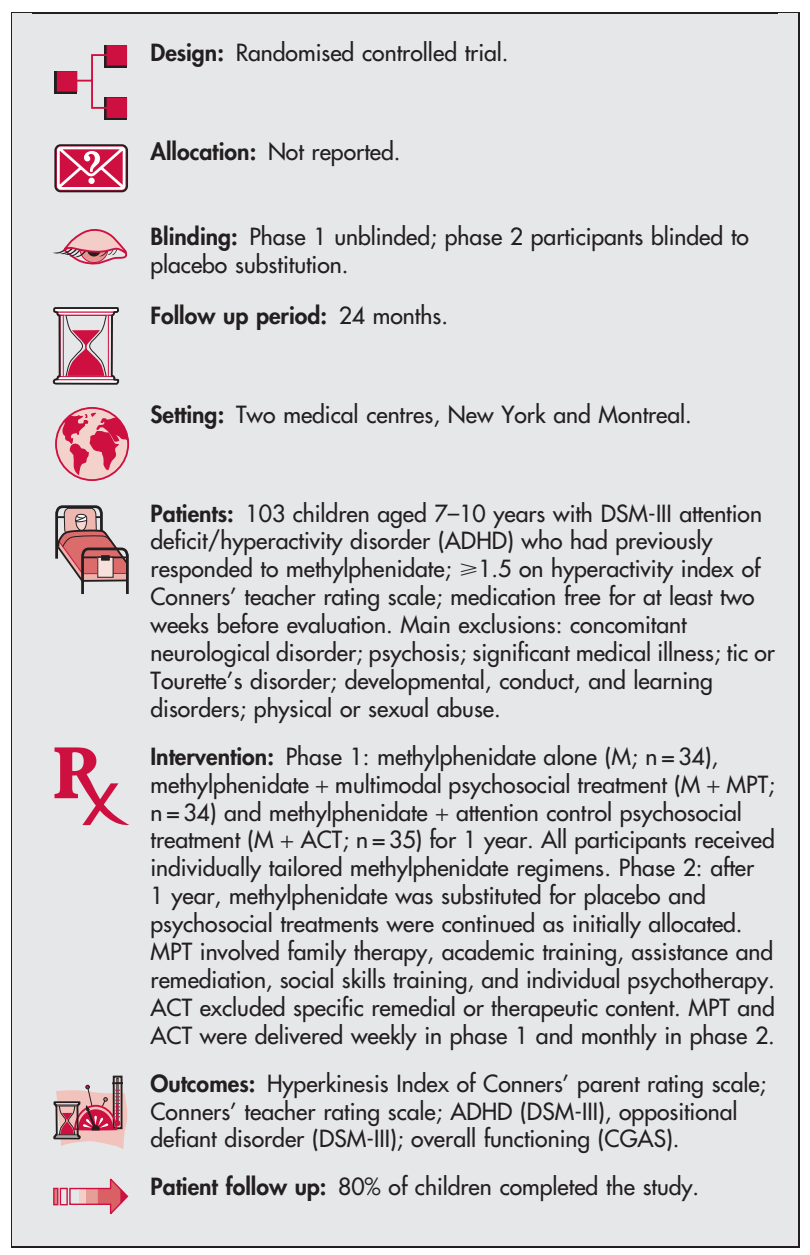

\section{MAIN RESULTS}

The paper did not present between group comparisons at the end of phase 1 . However, it concluded that there was no difference between treatments (see notes). In phase 2, after placebo substitution of methylphenidate, added MPT increased time to relapse compared with added ACT or no added psychosocial treatment, although results were not significant at the predefined 0.01 level (mean time to relapse: 8.6 days with $\mathrm{M}$ alone; 11.7 with $\mathrm{M}+\mathrm{ACT}$; 17.1 with $\mathrm{M}+\mathrm{MPT} ; \mathrm{p}<0.04$ for $\mathrm{M}+$ MPT versus $\mathrm{M}$ ).

\section{CONCLUSIONS}

There is no benefit from adding psychosocial treatment to methylphenidate in stimulant responsive children with ADHD.

For correspondence: Dr Ábikoff, NYU Child Study Centre, 215 Lexington Avenue, 13th Floor, New York, NY 10016, USA

Sources of funding: NIMH grant.

\section{NOTES}

Conclusions are limited by small sample size, so it is not possible to exclude clinically important effects of psychosocial treatment. Absolute results appeared to differ considerably among groups for some outcomes, but were not statistically compared (for example, prevalence of ADHD at 12 months varied from $6.5 \%$ to $16.7 \%$ ). The significance level was corrected to $\mathrm{p}=0.01$ for multiple analyses. Treatments were randomly allocated in blocks comprising four participants, so that group psychosocial treatment could be conducted.

\section{Commentary}

T his National Institute of Mental Health sponsored study is a valuable contribution to the field. Children ( $n=103$; age 7-9 years) were randomly assigned to methylphenidate only $(M)$, methylphenidate plus multimodal psychosocial treatment $(M+M P T)$, or methylphenidate plus attention control psychosocial treatment $(M+A C T)$. Before randomisation, all subjects showed a positive response to $M$ in a five week open label trial. Multiple outcomes were assessed every six months for two years. ${ }^{1}$

The paper did not specify a primary outcome measure. After six months, the effect size of $M$ was 1.4 on the parent rated Conners' Hyperkinesis Index and 2.5 on the teacher rated Conners' Hyperactivity scale. These large effects leave little room to detect additive effects of MPT on ADHD outcomes. Oppositional defiant disorder (ODD) symptoms are a plausible candidate for measuring the additive effects of MPT. However, only half of the study subjects met criteria for ODD. In addition, measures of ODD symptoms (clinician rated symptom count and the number of subjects moving from case to non-case in each group) did not capture the full dimensional range of severity.

Another design decision that hinders the interpretation of the study results was the inclusion of the $M+A C T$ group to control for the additional attention given to the M+MPT group. The study samples of 34 per group could detect an effect size in the range of 0.7 or 0.8 . By contrast, a sample size of 50 per group would be capable of detecting an effect size as low as 0.55 . The inclusion of the third study group all but guaranteed a finding of no difference between groups.

The findings of this study, which are consistent with the MTA study, ${ }^{2}$ may be somewhat discouraging to those who believe that medication combined with cognitive-behavioural interventions is inherently better than medication alone. The study design and measures presented in this report were unlikely to show a difference between groups because the large effect size of methylphenidate made it difficult to detect additive effects. One of the important contributions of this study is that it adds to the scant literature on the long term efficacy of stimulants in children with ADHD. Missing from this report is information on attrition, dosing, and adverse effects. Perhaps these findings will be presented in a separate report. Another clinical implication not stated in the report is the appropriateness of considering periodic withdrawal of stimulant medication to confirm ongoing need for treatment.

Lawrence Scahill, MSN, PhD Associate Professor of Nursing \& Child Psychiatry, Yale University, CT, USA

1 Klein RG, Abikoff $H$, Hechtman $L$, et al. Design and rationale of controlled study of long-term methylphenidate and multimodal psychosocial treatment in children with ADHD. J Am Child Adolesc Psychiatry 2004:43:792-801.

2 MTA Cooperative Group. A 14-month randomized clinical trial of treatment strategies for attention-deficit/hyperactivity disorder. Arch Gen Psychiatry 1999;56:1073-86. 